\title{
Asexual Plasmodium knowlesi Measurement
}

National Cancer Institute

\section{Source}

National Cancer Institute. Asexual Plasmodium knowlesi Measurement. NCI Thesaurus. Code C132417.

The determination of the amount of the organism assigned to the Plasmodium knowlesi species in the asexual phase in a biological sample. 